\title{
Pediatric Powered Mobility Training: Powered Wheelchair Versus Simulator-Based Practice
}

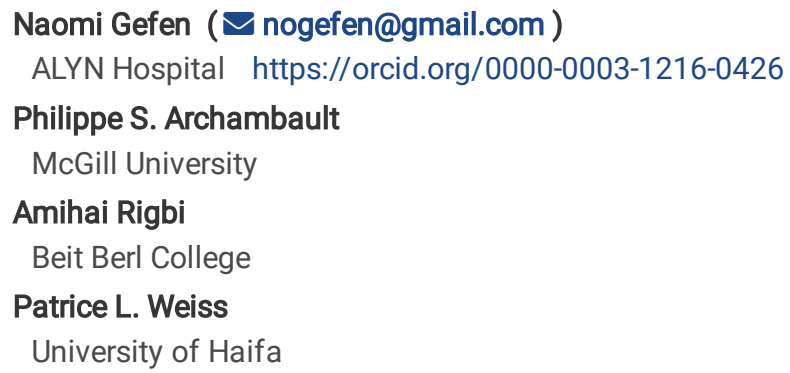

\section{Research}

Keywords: Pediatric powered mobility, simulator, PMP, ALP

Posted Date: November 23rd, 2021

DOI: https://doi.org/10.21203/rs.3.rs-1075055/v1

License: @ (i) This work is licensed under a Creative Commons Attribution 4.0 International License. Read Full License 


\section{Abstract}

Background: Many children with physical disabilities lack independent mobility. Powered wheelchair mobility can be a viable option, but users must be proficient drivers to ensure safety. To become a proficient driver, children need opportunities to practice. As is often the case, powered wheelchairs are scarce and direct therapy hours dedicated to powered mobility are often limited. Alternative options are needed to enable safe, unsupervised practice. Simulator-based learning has been shown to be an effective training method for powered mobility and other skill-based tasks for adults. The goal of this study was to compare two training methods of powered mobility, powered wheelchair practice (control group) versus simulator-based (experimental group) practice to determine whether simulation is a feasible and effective method for children and adolescents. The hypothesis was that children practicing on either modality at home supervised by a caregiver would similarly improve their powered mobility skills.

Method: Participants included 36 children and adolescents ( 23 males, 13 females) with cerebral palsy and neuromuscular diseases, aged 6-18 years (mean age: 10 y, 7 mo, SD: 3y, 7 mo). Data were collected and compared at baseline and after 12 weeks of home based practice of powered mobility via a powered wheelchair or a simulator. Thirty participants completed the study and were included in the analysis Powered mobility ability was determined by the Powered Mobility Program (PMP), the Israeli Ministry of Health's Powered Mobility Proficiency Test (PMPT) and the Assessment of Learning Powered Mobility use (ALP). Participants (21/30) were interviewed after study completion by an independent researcher concerning the child's and parent's experiences regarding practice time and user experience (e.g., satisfaction with training program, views of its importance).

Results: Both the powered wheelchair and simulator-based practice groups achieved significant improvement following the practice period as assessed by the PMP and PM-PT assessments, with no significant difference between them. A significant improvement was found in the ALP assessment outcomes for the powered wheelchair group only. All participants practiced for the required amount of time and both groups reported a similar user experience.

Conclusions: The results demonstrate that simulator-based practice is an effective training option for powered mobility for children aged 6-18 years old with physical disabilities. Simulator-based practice can provide a safe environment to practice driving skills that could endanger the child (e.g., out of doors). This is the first study, to our knowledge, that compares two different wheelchair training methods.

Trial Registration: The study protocol for this clinical trial was registered at ClinicalTrials.gov under the ID NCT04531488 and the title " Simulator Based Powered Mobility Training of Children With Special Needs "(Protocol ID: 004-17, URL: https://rb.gy/dfeslr).

\section{Background}

Self-locomotion is a major landmark in a child's development [1]. It facilitates exploration of the environment, peer interaction and communication while improving children's self-esteem and enhancing societal perception of their abilities [2-6]. When children with severe physical disabilities lack independent mobility, their general development is delayed and disparities with typically developing peers are widened [1].

Powered mobility (PM) has been shown to be a viable option for young children [6,7] provided they master a number of key skills including selfnavigation, spatial perception, and environmental awareness [1]. Safe wheelchair driving also requires a high level of attention, planning and judgment; thus most children become proficient drivers only after considerable practice (months and years) $[6,8,9]$. Practice options may be limited due to a lack of access to powered wheelchairs, limited therapist time and the need for constant supervision when practicing. Simulatorbased practice allows the user a safe and controlled environment to practice powered mobility and other related skills without constant supervision [10 ]. Technology platforms range from more immersive (e.g., Head mounted displays (HMD)) to less immersive (e.g., computer flat screens) devices that provide auditory or visual feedback. Some displays also provide haptic feedback [11,12]. Simulated practice has been shown to be an effective learning tool for adults who have a range of motor and cognitive impairments [13-15]. On the one hand, simulationbased practice may include the use of variations of a given route that require similar levels of skill to maintain the driver's engagement in the task, on the other hand, it often entails gradual increases in difficulty (e.g., narrowing of corridors) as skill level improves [16]. These and other assets known in the realm of virtual reality (e.g., virtual presence, addition of virtual avatars, documentation of results) [17] combine to encourage consideration of simulation as a candidate for powered mobility practice.

A range of powered mobility training methods was reviewed in a comprehensive survey of this field [18]. Although training on an actual powered wheelchair is the most commonly used technique, other methods such as simulators [13], smart robot wheelchairs [19] and modified "ride on cars" have also been investigated in intervention studies [18]. Regardless of the type of training method, several themes emerged: 1) the importance of training in a natural environment $[20,6] ; 2)$ the importance of parents and siblings in the training process $[21,22,23,24] ; 3)$ the use of game-like activities to motivate training $[25,26,27,28,29]$; and 4 ) the importance of training intensity and duration to improve powered mobility skills $[6,20,23]$. For example, Jones et al. [6] provided powered wheelchairs to children to practice in their natural environments (at 
home) for 12 months, supervised by their parents and Logan et al. [23] provided chairs to practice at home for 12 weeks with a goal of enabling daily practice sessions of 20-30 minutes. In both studies children were able to incorporate powered mobility into their natural environment and progress in their driving skills.

The majority of studies investigating pediatric powered wheelchair training were carried out on very young children, with very few focusing on older children who have not yet mastered independent driving. A recent study [30], did evaluate an intensive three-week powered mobility summer camp for children with cerebral palsy aged 11-15 years; all 24 participants significantly improved their PM skills as assessed by the Powered Mobility Program (PMP) and Assessment of Learning Powered Mobility tool (ALP).

A scoping review [10] examined different aspects of powered mobility training with a simulator focusing on the sense of presence, user interaction and perception as well as on the effectiveness of this type of training. All of the reviewed studies reported improvement in driving skills as measured in both simulator and real-world tasks, thus supporting the ability to transfer skills obtained via simulator practice. Increased training duration with the simulator was shown to improve driving skills [10].

Both systematic reviews $[10,18]$ focused on either PM training or the use of a simulator. To date, simulator-based training has not been compared directly to training of children on a powered wheelchair. Another difference between the current study and other powered mobility studies is that it was a remote (non clinical)-based practice program, i.e, children practiced at home and, in some cases also at school, intensifying their practice time. Children that practiced at home had the benefit of a caregiver dedicated only to them, without having to "compete" for the therapist's time. The families were highly motivated to practice and help their child become a proficient driver.

The main objective of this study was to assess simulator-based powered mobility training and to determine its effectiveness in comparison to powered mobility training. The hypothesis was that children practicing on a chair or via a simulator at home would improve their powered mobility skills significantly, without a significant difference between training methods. This was accomplished by comparing the pre- and posttraining PMP, Israeli Ministry of Health's Powered Mobility Proficiency Test (PM-PT) and ALP assessment results in two equivalent groups of children (powered wheelchair versus simulator).

\section{Methods}

\section{Study Design}

This study used a parallel pragmatic design (quasi-randomization) where participants were recruited from a list of children waiting to be provided with a powered wheelchair from the ALYN Hospital PM lending program. The waiting list that was in place at the start of the study (May 2018) was continually updated with additional children until the study end date (June 2020). Since the effectiveness of simulator practice was unproven, i.e., there was no assurance that it would lead to proficiency in wheelchair driving, the hospital's Institutional Review Board required that participants in the simulator group be assigned from the bottom half of the waiting list. In contrast, participants in the control group (training via an actual wheelchair that was considered to be "standard service procedures") were assigned from the top half of the waiting list. Randomization for each group thus occurred within each half of the waiting list. Since there were no differences in age, gender, and other demographics of the participants in the two halves, this allocation scheme merely ensured that powered wheelchair training was given on a "first in-first out" basis. Moreover, a commitment was made to the parents of all children who practiced on the simulator that a powered wheelchair would be offered upon completion of the study if proficiency was not obtained. All children and parents spoke with the research coordinator and first author prior to giving their informed consent to participate in the study.

\section{Participants}

Thirty-six children (23 males, 13 females), aged 6-18 years (mean age: 10yrs, 7mon; SD: 3yrs, 7mon), with cerebral palsy and other neuromuscular diseases were recruited through the ALYN Hospital lending program for powered wheelchairs. All participants were able to control the chair with a joystick. Participants were excluded if they were younger than six years of age; had unstable medical conditions that prevented them from participating in powered mobility training (e.g., epilepsy); were able to operate a powered wheelchair only via ability switches or a scanning device or had visual acuity or field deficits that interfered with their ability to view and respond to stimuli presented on a screen or while driving.

\section{Outcome Measures and Screening Classifications}

Demographic, Functional and Accessibility Questionnaire was completed by a parent and therapist in the community to gather information regarding the participant's diagnosis, medical and developmental history, functional level, hand dominance, mobility and transportation. Information regarding home/school accessibility and prior experience with powered mobility was provided by the child's therapist after a home visit. All information was provided before study commencement. 
User experience questionnaire was completed by a parent via a telephone interview after the conclusion of the program to provide information regarding practice time, overall satisfaction, "cyber-sickness" (for the simulator group only), social validity and parental perspective of practice progress. The 7-item questionnaire used a five-point Likert scale, where " 1 " denoted strong disagreement and " 5 " denoted strong agreement in all items except the item about recommended practice time. For that item, "1" denoted practiced less than the recommended time, " 3 " practiced for the recommended time and " 5 " practiced for more than the recommended time.

\section{Screening Classifications}

These were completed by a therapist as part of the demographic, functional and accessibility questionnaire.

Level of Sitting Scale (LSS)[31] uses an eight-point scale to rate how much support a child needs to sit independently.

Gross Motor Function Classification Scale Expanded and Revised (GMFCS-ER) uses a five-point scale to rate the gross motor level of children with CP aged 2-18 years [32,33].

Manual Ability Classification Scale (MACS) uses a five-point scale to rate the child's ability to handle objects during daily activities. It can be used for children with CP aged 4-18 years [34].

Communication Function Classification Scale (CFCS) uses a five-point scale to rate the everyday communication abilities with familiar and unfamiliar partners of children with CP, aged 2-18 years [35].

Brooke Upper Limb Function Scale is a six-point scale that describes the functional level of the upper extremities of children with Duchenne and other neuromuscular diseases [36].

Vignos Lower Limb Function Scale is a 10-point scale that categorizes the walking abilities of children with Duchenne muscular dystrophy and other neuromuscular diseases [37].

\section{Primary outcome measures of powered mobility proficiency}

Powered Mobility Program (PMP) [38] is a three-part evaluation of up to 34 driving skills that measures a child's ability to drive a powered wheelchair in and outdoors. Skills include, for example, the ability to drive through doors, enter a room, drive down a corridor, drive up and down an outside slope. Each item is scored on a 6-point scale (0-5) and relates to the level of physical and verbal support that the child needs to complete a skill [38]. Median scores were calculated to achieve a final score. The current study used a reliable and valid Hebrew translation of the PMP [39].

Assessment of Learning Powered Mobility Use (ALP) $[39,40]$ uses an eight-point scale to assess five different cognitive and emotional aspects of using powered mobility for children as young as twelve months and up until adulthood. Aspects include, for example, the ability to interact with the chair, multi-tasking while driving, and a reaction to movement. Median scores were calculated for the child's PM level. The current study used a reliable and valid Hebrew translation of the ALP [39].

Israel Ministry of Health PM Proficiency Test (PM-PT) [39] is a three-point scale (pass=5, needs practice=3, fail=1) Hebrew-language test with seven items that assesses the user's powered wheelchair skills. Skills include, for example, the ability to stop voluntarily when asked to, to drive around obstacles, drive through a doorway, and drive up to 25 meters. A person is considered a competent PM driver if they are able to pass each item in a consistent manner four out of five times. It is used by therapists to determine who is entitled to government funding of a powered wheelchair. Median scores were calculated as the final score. The PM-PT is reliable and valid [39].

The three PM assessments were administered prior to and following the intervention. The PM-PT was chosen because it is a short evaluation that is widely used in Israel to test wheelchair driving proficiency. The PMP and ALP were chosen because they are the leading evaluations for pediatric powered mobility. All three PM assessments were recently shown to be reliable and valid for the study age group [39]. Due to the ordinal scale of the items composing these measures, total scores were achieved by calculating the medians across the participants.

\section{Instruments}

Powered Wheelchairs for the control group were provided through the ALYN Lending Program. Chairs were selected from a pool of 21 loan units with a variety of characteristics (size, tilt, recline) and included the most commonly used models (e.g. Invacare, Pride Mobility, Permobil). Each chair was configured optimally for the participant's use.

The McGill Immersive Wheelchair Simulator (MiWe) [13-15] was developed with the Unity 3D graphics language to compare real power mobility movement to that of the simulator and as a clinical assessment and practice tool for adults and to examine upper extremity reaching during 
powered mobility. The MiWe runs on a Windows computer, with an operating system of at least Windows 7, with a graphics card of at least 1 GB of video memory and able to run DirectX 11. It displays on a standard screen. The user operates a joystick to interact with a series of six simulated environments via a first person, non-stereoscopic viewpoint. Joystick actions, the simulated wheelchair trajectory, collisions and tasks duration are recorded. Each environment has three levels differing in the number of tasks, obstacles and the time allotted per task. The tasks were based on the Wheelchair Skills Test from Dalhousie University [41]. The environments and tasks are:

1. Elevator - navigate to an elevator, press outside button, enter elevator, press internal button, and exit elevator.

2. Van - navigate in an outside parking lot to a van and then up a van ramp, position wheelchair in the van, and exit van via ramp.

3. Shopping Mall - enter mall, navigate to vending machine and then to trash bin, and exit mall.

4. Supermarket - enter supermarket, navigate to locate and retrieve food from shelves, pay for purchased item, and exit supermarket.

5. Street Crossing - navigate to crosswalk, press street crossing button, cross street to pedestrian island, press a second street crossing button, and cross to other side of street.

6. Bathroom - navigate to bathroom, enter bathroom, position wheelchair near toilet, navigate to sink, exit bathroom, and navigate to the exit sign.

7. Day of errands- all environments were merged to provide a longer route

The MiWe was modified for use at ALYN Hospital with children (MiWe-C) [16]; two routes at ALYN Hospital were added to be used for testing purposes, and a Hebrew language interface was provided. Photos and screenshots in Figure 1 show several example views of the route.

\section{Procedures}

During the first assessment, the participants were evaluated to determine their optimal seating level and access site for wheelchair activation. Participants in both groups were fitted with a powered wheelchair and seating supports (if required) by a seating expert from the ALYN lending program. The positioning of the participants in the experimental group was documented to ensure that they would sit as similarly as possible during the pre- and post-intervention evaluations. Participants from both groups drove the chair throughout a spacious demonstration center for about 15 min before the initial PM evaluation. They then drove the chair for 30-60 minutes along one of the two test routes, which entailed maneuvering it through ALYN Hospital hallways and doorways, driving to specified destinations, stopping upon request and driving up an incline. An experienced occupational therapist walked next to child to ensure safety. The extent of provided help was recorded as part of the PMP evaluation. Driving along the route was videotaped by a parent or second therapist for later analysis by two expert raters [39]. The control group then left with a loaner chair which they kept until the end of the study. Experimental group participants were provided with a laptop, joystick and the MiWe-C program and were instructed on how to use the simulator for practice at home or school. Both the control and experimental groups were instructed to practice at least four times a week for a minimum of 20 minutes. Parents in both groups were given instructions regarding their child's practice training sessions, encouraging them to adopt a family-centered approach. [42]

Participants returned to ALYN Hospital for a post-training evaluation after 12 weeks. They physically drove along one of two, randomly selected test routes. The simulator group also drove the same route but within the simulated environment. Videotapes of each evaluation were later analyzed by one of two raters using the PMP, ALP and PM-PT [39]. Participants in either group who demonstrated proficiency were referred to the Ministry of Health for procurement of their own chair. Children who did not demonstrate proficiency were provided additional practice opportunities; these additional data are not included in the current analysis. A user experience questionnaire was completed via a telephone interview after the conclusion of the program.

\section{Data Analysis}

A power analysis specifying a medium effect size (cutoff: $r=0.30$ ), $\alpha=0.05$ and $1-\beta=0.80$ revealed that a target sample size of 20 participants per group was required in order to achieve the recommended statistical power. Descriptive statistics included counts and percentages in the case of categorical variables, medians and inter-quartile ranges in the case of ordinal variables, means and standard deviations in the case of continuous variables. Group differences were determined by the Pearson Chi-square test and the Fisher exact test for the categorical variables. The Mann-Whitney test or independent samples t-tests were used to determine differences between the groups for the ordinal and the continuous variables respectively between pre- and post-practice. Means and standard deviations were used to document user experience.

Effect size was calculated by converting Z statistics to Pearson's $r$ and interpreted as follows [43]: small $(r=0.10)$; medium ( $r=0.30)$; large $(r>0.50)$. The minimal clinically important differences (MCID) were calculated for all three assessments (PMP, ALP and PM-PT) by comparing their SEM (SD/ $\sqrt{ }$ (count (post-pre)) to the mean of the pre-post difference. If the SEM was less than the mean, the pre-post change was considered to be clinically significant [44-47]. 
All analyses were carried out using SPSS software, version 25. Statistical significance was set to $a \leq 0.05$. The pre-intervention video recordings were analyzed by two raters using the PMP, ALP and PM-PT. Video recordings of the post-intervention were analyzed by one rater using the PMP, ALP and PM-PT [39]. The raters were blinded to group allocation.

\section{Results}

The Consort diagram, shown in Figure 2, indicates that 36 participants were recruited from an initial pool of 73 children with 18 allocated to each group. Two participants in each group did not complete training due to behavioral issues and a lack of motivation to practice. In addition, two participants in the simulator group completed the simulator training but were unavailable for post-testing due to Covid-19-related limitations. Thus, data from a total of 30 participants were analyzed.

\section{Participant Characteristics}

Table 1a summarizes the background characteristics of the participants including their diagnosis, functional level, demographic information, manual wheelchair level. Table 1b summarizes their baseline outcome measure scores. There were no statistically significant differences between the groups apart from practice location where a greater percentage of children in the control group practiced both at home and school, compared to the simulator group. The descriptive analysis revealed that the simulator group was characterized by a greater proportion of children who can propel a manual WC.

Analysis of the PMP assessment results (Table 2, Figure 3a) revealed a significant effect for practice overall $(p<0.001)$ with a medium effect size ( $r=-0.47)$ and a significant effect for practice within each group separately (Simulator practice: $p=0.04 ;$ Powered mobility: $p=0.003)$ with medium and large effect sizes ( $r=-0.39, r=-0.52$, respectively). The overall between-group effect was not significant ( $p=0.95)$ and neither were the effects between the groups at each time-point (pre-practice: $p=0.22$; post-practice: $p=0.47$ ).

Analysis of the ALP assessment results (Table 2, Figure $3 b)$ revealed a significant effect for practice overall $(p=0.02)$, with a medium effect size $(r=-0.30)$ and a significant effect for practice within the powered mobility group only (Simulator practice: $p=0.33 ; P o w e r e d$ mobility: $p=0.03)$ with medium effect size $(r=-0.39)$. The overall between-group effect was not significant $(p=0.38)$ and neither were the effects between the groups at each time-point (pre-practice: $p=0.22$; post-practice: $p=0.67$ ).

Analysis of the PM-PT assessment results (Table 2, Figure 3c) revealed a significant effect for practice overall ( $<<0.001$ ), with a medium effect size $(r=-0.46)$ and a significant effect for practice within each group separately (Simulator practice: $p=0.01 ; P$ owered mobility: $p=0.007)$ with medium effect sizes ( $r=-0.45, r=-0.47$, respectively). The overall between-group effect was not significant (Mann-Whitney test: $p=0.45)$ and neither were the effects between the groups at each time-point (pre-practice: $p=0.58$; post-practice: $p=0.67$ ).

The MCID was calculated for all three assessments (PMP, ALP and PM-PT) as indicated in the data analysis section. The PMP (mean=0.63; $\mathrm{SEM}=0.15$ ) and ALP (mean= 0.52; SEM=0.24) demonstrated significant MCIDs. The PM-PT did not reach significant MCID (mean=0.55; SEM=0.68).

Twenty-one parents participated in a telephone interview (15/16 powered wheelchair group; 5/14 simulator group) to document user experience during the home-based practice period. Items 1-6 showed that both parents and children enjoyed the practice and understood its importance as a way to achieve independent mobility, as shown in Table 3. Both groups practiced the recommended amount of time or more (Item 7- "3" recommended amount of time). The proportion of parents (36\%) in the simulator group who responded was considerably lower than the powered wheelchair group (84\%).

Taken together, these results support the hypothesis that practice improves powered mobility driving skills regardless of training mode.

\section{Discussion}

The goal of this study was to determine whether simulator-based powered mobility practice is a viable training option for children with mobility impairments. The findings revealed that participants as young as six years improved their actual powered wheelchair driving skills over a 12week program regardless of the practice method. In previous studies of powered mobility training, only younger children were assessed, the training period was 6-12 months, and only a single training method (conventional powered mobility, simulator-based training, "ride-on" cars, smart wheelchairs) was tested $[6,18]$. We note that a relatively short training period (12 weeks) was used in the current study since the goal was to compare training methods and the ability to progress in powered mobility skills rather than ensuring that they became proficient drivers (although this was an important secondary aim that was achieved by $27 \%$ of the children in the control group and $30 \%$ of the children in the simulator group). Thus, we used a training period that is considerably shorter than reported in a retrospective study of 80 children who participated in a powered wheelchair lending program [48]; sixty-five percent (52) of the participants became proficient drivers following a mean 
training period of 33 weeks (range $=21-47$ weeks). Other studies [6] provided up to one year of training. Both studies provided home-based practice with parents being responsible for ensuring sufficient practice opportunities.

In two comprehensive systematic reviews [10,18], different powered wheelchair training methods were evaluated for children with and without disabilities. The studies described the use of powered wheelchairs, smart navigation, virtual reality programs and physical powered wheelchair simulators; each investigated a single training method. The direct comparison of two different training methods, as carried out in the current study, provides, for the first time, evidence that confirms the feasibility and effectiveness of using a simulator for powered wheelchair practice.

The ALP results showed a significant improvement in overall driving skills only in the powered mobility training group. This may be explained by the fact that the ALP assesses, in part, interaction with the chair, attention and the ability to multitask; these specific skills are not practiced while training on the simulator. Moreover, simulator-based practice does not provide motion feedback or 3D spatial interaction that a powered wheelchair does; practicing on a powered wheelchair exposes the child to independent movement that they may otherwise lack. This highlights the fact that each type of practice method has advantages and limitations that are important to recognize when considering their suitability as training options. For example, using a simulator can be safer, in contrast to the constant supervision a child needs when beginning to practice on a powered wheelchair. The chair and simulator can be used simultaneously to increase and enhance practice time and opportunities. A child practicing at school on a powered chair can use a simulator at home for additional practice time. If a child lives in an inaccessible apartment and is in the process of moving to a more suitable environment, a simulator can be used to expedite the practice process rather than waiting until the move. Some younger children or children with intellectual and developmental disorders may be intimidated by using a large powered chair especially when it is moving. In such cases, a simulator would help them gradually get used to powered mobility.

Transfer of powered mobility skills from simulator-based practice to real world skills has been addressed previously. Linden et al. [25] studied 28 typically developing children who were evaluated on a powered wheelchair and their driving skills were observed. Fourteen, randomly assigned children underwent simulator-based practice for eight 30-minute sessions; the remaining children had no practice. The experimental group significantly improved their driving skills compared to the no-intervention control group. This provides some evidence for the transfer of skills following practice with a two-dimension powered mobility computer simulation to the three-dimension task of driving a powered wheelchair. Further research is needed.

Another positive aspect of simulator-based practice is the ability to practice outside driving skills in the safety of the child's home. Crossing the street, avoiding potholes and other barriers, or driving in non-idyllic weather conditions are all challenging when a child practices on a powered wheelchair. Being able to master these skills in a more controlled environment is important. Morere et al. [49] assessed 12 children and young adults with cerebral palsy, eight proficient indoor drivers and four novice drivers, who practiced outdoor driving skills on a simulator. Eleven of the twelve participants improved their general driving skills (indoor and outdoor) and five out of the eight proficient indoor drivers were then able to pass their outdoor driving test, showing that a simulator can be used to master specific challenges as well as for basic training.

The simulator used in the current study (MiWi-C) is a PC-based program that uses a USB joystick. Having a simulator that can be used for practice in the community rather than requiring specific laboratory conditions is a considerable advantage. The majority of simulators used in previous studies, as noted in Arlati et al.'s [10] and Lam et al.'s [50] systematic reviews, were laboratory-based, making them relevant primarily for research rather than as clinical or home-based tools that can be implemented into a daily routine. When trying to master a skill, intensive practice has been shown to improve performance [10]. Having a simulator at home or school enables the child to achieve more practice time.

Home-based therapy is often used in rehabilitation. Many studies have shown the benefits and effectiveness of home-based, caregiver directed rehabilitation in different populations including stroke survivors [51, 52], spinal cord injury [53], cystic fibrosis [54], and cerebral palsy [55]. Similar to the protocol used in the current study, these programs were based on caregiver directed rehabilitation and promoted a family centered approach. Home-based rehabilitation appears to increase and prolong therapy dosage after discharge and to take advantage of technology provided it is adapted to the home environment.

The results of the current study were reported to the Israeli Ministry of Health who have now adopted a policy of fully funding powered wheelchairs for children who show proficiency in a real powered wheelchair after training solely on a simulator. In countries where proficiency is a criterion for funding a powered wheelchair, having additional, affordable practice options may help more children become proficient powered wheelchair drivers, thereby providing participation options for children with disabilities.

\section{Limitations}

There were several limitations in this study. Due to the simulator's current configuration, this study sampled only children who had the ability to activate their chair with a joystick; children whose limitations would have necessitated the use of ability switches or a scanner were thus not included. Moreover, the results of this study are not based on a purely probabilistic sample, due to ethical considerations when using recruiting from a waiting list that had to be prioritized according to first-in/first-out; hence some caution concerning generalizability in required.

Page $7 / 17$ 
An important limitation is related to the study's sample size. The sample size analysis performed prior to the study indicated a target of 40 participants. After two years of recruitment only 36 suitable participants joined the study and only 30 completed the protocol for the reasons described above. Regrettably, due to COVID 19 lockdown restrictions beginning in June 2020, hospital visits related to experimental data collection were cancelled. Since the current study required an essential in-person, on-site series of evaluations, it was not possible to recruit additional participants to replace drop-outs. The lockdown also limited the collection of post-intervention data (which also required in-person, on-site presence) from two participants from the simulator group. We note that although the sample size was lower than that recommended in the original power calculation, it is comparable to those of other recent studies in the field of powered mobility $[5,6,30,56)$. Hence, while we acknowledge this limitation, we contend that the results do contribute to the evidence supporting the use of home-based simulator practice to enhance powered mobility proficiency. Nevertheless, generalization of the results should remain cautious.

Another limitation is the lack of objective monitoring of practice time which was mandated to be at least four times a week for 20 minutes. Although parents reported that their children practiced more than the mandated time, direct documentation of practice time throughout the study would have provided a more objective measure. We note that considerably more parents of children that practiced on the powered wheelchair (15/16) responded to the questionnaire as compared to the simulator group (5/14). This may be indicative of differences in parental attitude towards the two practice modes. Some of parents from the simulator group that did not participate in the interview (64\%) may have preferred not to speak negatively about their child's experience and hence refrained from partaking in the interview, as documented in other studies [57]. This potential effect should be studied further.

Future studies should also address whether children with intellectual and developmental disorders could benefit from practicing on a powered wheelchair simulator and if the simulator could be set up for children accessing the chair via a scanner or switches. Another study should also address a hybrid approach of powered wheelchair practice at school and simulator practice at home regarding progress and proficiency.

\section{Conclusions}

Powered mobility simulators provide children and adults practice opportunities that may not be available to them otherwise, due to the cost of a powered chair for training, therapy time and safety concerns. The demonstrating of additional feasible training methods such as a simulator to learn powered mobility skills and to achieve proficiency may be beneficial for healthcare systems where powered wheelchair proficiency is mandatory before funding a chair.

\section{Declarations}

\section{Ethics approval and consent to participate}

Ethics approval was granted by the Internal Review Board at the ALYN Hospital (approval number 004-17). A parent of each participant provided written informed consent after the study procedure had been fully explained; the children gave their verbal assent. The study protocol for this clinical trial was registered at ClinicalTrials.gov under the ID NCT04531488 and the title " Simulator Based Powered Mobility Training of Children With Special Needs "(Protocol ID: 004-17, URL: https://rb.gy/dfes/r). The study was conducted between May 2018 (start of data collection) and July 12, 2020 (end of both the data collection and active phase).

\section{Competing interests}

The authors have no competing interests to declare.

\section{Consent for publication}

Not applicable

\section{Availability of data and materials}

The data generated during and/or analyzed during the current study are not publicly available due to policy restrictions dictated by the IRB, but are available from the corresponding author on reasonable request.

\section{Funding}

This study was funded by ALYN Hospital. The first author received a scholarship from the University of Haifa for her doctoral research which includes the reported study.

\section{Authors' contributions}


NG contributed to this work including: the conception, design of the work, the acquisition, analysis, and interpretation of data, and wrote the manuscript.

AR was responsible for data analysis, interpretation of the results and manuscript revision

PA conception of the simulator, design of the work, manuscript revision.

PLW contributed to the conception, design of the work, acquisition, interpretation and manuscript revision.

\section{Acknowledgements}

We would like to thank the children and families that participated in the study. We would also like to thank Osnat Yaniv and Avigail Haimovski for their help in assessing the children. This study was carried out as part of the first author's doctoral dissertation.

\section{References}

1. Anderson DI, Campos JJ, Witherington DC, Dahl A, Rivera M, He M, Uchiyama I, Barbu-Roth M. The role of locomotion in psychological development. Frontiers in psychology. 2013 Jul 23;4:440.

2. Rosen L, Plummer T, Sabet A, Lange ML, Livingstone R. RESNA position on the application of power mobility devices for pediatric users. Assistive Technology. 2018 Mar 26:1-9.

3. Guerette P, Furumasu J, Tefft D. The positive effects of early powered mobility on children's psychosocial and play skills. Assistive Technology. 2013 Mar 1;25(1):39-48.

4. Kenyon LK, Mortenson WB, Miller WC. 'Power in Mobility': parent and therapist perspectives of the experiences of children learning to use powered mobility. Developmental Medicine \& Child Neurology. 2018 Oct;60(10):1012-7.

5. Butler C, Okamoto GA, McKay TM. Powered mobility for very young disabled children. Developmental Medicine \& Child Neurology. 1983 Aug;25(4):472-4.

6. Jones MA, McEwen IR, Neas BR. Effects of power wheelchairs on the development and function of young children with severe motor impairments. Pediatric Physical Therapy. 2012 Jul 1;24(2):131-40

7. Galloway JC, Ryu JC, Agrawal SK. Babies driving robots: self-generated mobility in very young infants. Intelligent Service Robotics. 2008 Apr 1;1(2):123-34.

8. Livingstone R, Paleg G. Practice considerations for the introduction and use of power mobility for children. Developmental Medicine \& Child Neurology. 2014 Mar;56(3):210-21.

9. Nilsson L, Eklund M, Nyberg P, Thulesius H. Driving to learn in a powered wheelchair: the process of learning joystick use in people with profound cognitive disabilities. American Journal of Occupational Therapy. 2011 Nov 1;65(6):652-60.

10. Arlati S, Colombo V, Ferrigno G, Sacchetti R, Sacco M. Virtual reality-based wheelchair simulators: A scoping review. Assistive Technology. 2019 Jan 7.

11. Gandhi RD, Patel DS. Virtual reality-opportunities and challenges. Virtual Reality. 2018 Jan;5(01).

12. Deutsch JE, Boian RF, Lewis JA, Burdea GC, Minsky A. Haptic effects modulate kinetics of gait but not experience of realism in a virtual reality walking simulator. In2008 Virtual Rehabilitation 2008 Aug 25 (pp. 36-40). IEEE.

13. Bigras C, Kairy D, Archambault PS. Augmented feedback for powered wheelchair training in a virtual environment. Journal of NeuroEngineering and Rehabilitation. 2019 Dec;16(1):1-2.

14. Archambault PS, Tremblay S, Cachecho S, Routhier F, Boissy P. Driving performance in a power wheelchair simulator. Disability and Rehabilitation: Assistive Technology. 2012 May 1;7(3):226-33.

15. Tao G, Archambault PS. Powered wheelchair simulator development: implementing combined navigation-reaching tasks with a 3D hand motion controller. Journal of NeuroEngineering and Rehabilitation. 2016 Dec;13(1):1-3.

16. Gefen N, Rigbi A, Archambault PS, Weiss PL. Comparing children's driving abilities in physical and virtual environments. Disability and Rehabilitation: Assistive Technology. 2021 Aug 18;16(6):653-60.

17. Parsons TD, Rizzo AA, Rogers S, York P. Virtual reality in paediatric rehabilitation: a review. Developmental Neurorehabilitation. 2009 Jan 1;12(4):224-38.

18. Kenyon LK, Hostnik L, McElroy R, Peterson C, Farris JP. Power mobility training methods for children: a systematic review. Pediatric Physical Therapy. 2018 Jan 1;30(1):2-8.

19. Marchal-Crespo L, Furumasu J, Reinkensmeyer DJ. A robotic wheelchair trainer: design overview and a feasibility study. Journal of NeuroEngineering and Rehabilitation. 2010 Dec;7(1):1-2. 
20. Jones MA, McEwen IR, Hansen L. Use of power mobility for a young child with spinal muscular atrophy. Physical therapy. 2003 Mar $1 ; 83(3): 253-62$.

21. Dunaway S, Montes J, O'Hagen J, Sproule DM, Vivo DC, Kaufmann P. Independent mobility after early introduction of a power wheelchair in spinal muscular atrophy. Journal of child neurology. 2013 May;28(5):576-82.

22. Huang HH. Perspectives on early power mobility training, motivation, and social participation in young children with motor disabilities. Frontiers in psychology. 2018 Jan 9;8:2330.

23. Logan SW, Lobo MA, Feldner HA, Schreiber M, MacDonald M, Winden HN, Stoner T, Galloway JC. Power-up: exploration and play in a novel modified ride-on car for standing. Pediatric Physical Therapy. 2017 Jan 1;29(1):30-7.

24. Butler $\mathrm{CH}$, Okamoto GA, McKay TM. Motorized wheelchair driving by disabled children. Archives of physical medicine and rehabilitation. 1984 Feb 1;65(2):95-7.

25. Linden MA, Whyatt C, Craig C, Kerr C. Efficacy of a powered wheelchair simulator for school aged children: A randomized controlled trial. Rehabilitation psychology. 2013 Nov;58(4):405.

26. Huang WP, Wang CC, Hung JH, Chien KC, Liu WY, Cheng CH, Ng HH, Lin YH. Joystick-controlled video console game practice for developing power wheelchairs users' indoor driving skills. Journal of physical therapy science. 2015;27(2):495-8.

27. Chen X, Ragonesi C, Galloway JC, Agrawal SK. Design of a robotic mobility system with a modular haptic feedback approach to promote socialization in children. IEEE transactions on haptics. 2013 Aug 15;7(2):131-9.

28. Inman DP, Loge K, Cram A, Peterson M. Learning to drive a wheelchair in virtual reality. Journal of Special Education Technology. 2011 Sep;26(3):21-34.

29. Adelola IA, Cox SL, Rahman A. Virtual environments for powered wheelchair learner drivers: Case studies. Technology and Disability. 2009 Jan 1;21(3):97-106.

30. Rosenberg L, Maeir A, Gilboa Y. Evaluating a Therapeutic Powered Mobility Camp for Children with Severe Cerebral Palsy: Évaluation d'un camp thérapeutique de mobilité motorisée pour les enfants atteints de paralysie cérébrale grave. Canadian Journal of Occupational Therapy. 2021 Aug 26:00084174211034938.

31. Fife SE, Roxborough LA, Armstrong RW, Harris SR, Gregson JL, Field D. Development of a clinical measure of postural control for assessment of adaptive seating in children with neuromotor disabilities. Physical Therapy. 1991 Dec 1;71(12):981-93

32. Rosenbaum PL, Palisano RJ, Bartlett DJ, Galuppi BE, Russell DJ. Development of the gross motor function classification system for cerebral palsy. Developmental Medicine \& Child Neurology. 2008 Apr;50(4):249-53.

33. Bodkin AW, Robinson C, Perales FP. Reliability and validity of the gross motor function classification system for cerebral palsy. Pediatric Physical Therapy. 2003 Dec 1;15(4):247-52.

34. Eliasson AC, Krumlinde-Sundholm L, Rösblad B, Beckung E, Arner M, Öhrvall AM, Rosenbaum P. The Manual Ability Classification System (MACS) for children with cerebral palsy: scale development and evidence of validity and reliability. Developmental medicine \& child neurology. 2006 Jul;48(7):549-54.

35. Hidecker MJ, Paneth N, Rosenbaum PL, Kent RD, Lillie J, Eulenberg JB, CHESTER, JR KE, Johnson B, Michalsen L, Evatt M, Taylor K. Developing and validating the Communication Function Classification System for individuals with cerebral palsy. Developmental Medicine \& Child Neurology. 2011 Aug;53(8):704-10.

36. Brooke MH, Griggs RC, Mendell JR, Fenichel GM, Shumate JB, Pellegrino RJ. Clinical trial in Duchenne dystrophy. I. The design of the protocol. Muscle \& Nerve: Official Journal of the American Association of Electrodiagnostic Medicine. 1981 May;4(3):186-97.

37. Florence JM, Pandya S, King WM, Robison JD, Signore LC, Wentzell M, Province MA. Clinical trials in Duchenne dystrophy: standardization and reliability of evaluation procedures. Physical therapy. 1984 Jan 1;64(1):41-5.

38. Furumasu J, Guerette P, Tefft D. The development of a powered wheelchair mobility program for young children. Technology and Disability. 1996 Jan 1;5(1):41-8.

39. Gefen N, Rigbi A, Weiss PL. Reliability and Validity of Pediatric Powered Mobility Outcome Measures. Disability and Rehabilitation: Assistive Technology. 2020 Sep 11:1-6.

40. Svensson E, Nilsson L. Inter-rater reliability of the assessment of learning powered mobility use, version 2.0, when applied with children and adults engaged in Driving to Learn in a powered wheelchair. Australian Occupational Therapy Journal. 2021 Apr;68(2):115-23.

41. Kirby RL, Dupuis DJ, MacPhee AH, Coolen AL, Smith C, Best KL, Newton AM, Mountain AD, MacLeod DA, Bonaparte JP. The wheelchair skills test (version 2.4): measurement properties. Archives of physical medicine and rehabilitation. 2004 May 1;85(5):794-804.

42. Litterini AJ, Wilson CM. Caregivers in Patient-and Family-Centered Care. InPhysical Activity and Rehabilitation in Life-threatening IIIness 2021 May 4 (pp. 61-74). Routledge.

43. Field A. Discovering statistics using SPSS. Sage publications; 2009 Jan 21.

Page $10 / 17$ 
44. Musselman KE. Clinical significance testing in rehabilitation research: what, why, and how? Physical Therapy Reviews. 2007 Dec $1 ; 12(4): 287-96$.

45. Copay AG, Subach BR, Glassman SD, Polly Jr DW, Schuler TC. Understanding the minimum clinically important difference: a review of concepts and methods. The Spine Journal. 2007 Sep 1;7(5):541-6.

46. Rai SK, Yazdany J, Fortin PR, Aviña-Zubieta JA. Approaches for estimating minimal clinically important differences in systemic lupus erythematosus. Arthritis research \& therapy. $2015 \mathrm{Dec} ; 17(1): 1-8$.

47. Norman GR, Sloan JA, Wyrwich KW. Interpretation of changes in health-related quality of life: the remarkable universality of half a standard deviation. Medical care. 2003 May 1:582-92.

48. Gefen N, Rigbi A, Weiss PL. Predictive model of proficiency in powered mobility of children and young adults with motor impairments. Developmental Medicine \& Child Neurology. 2019 Dec;61(12):1416-22.

49. Morère Y, Bourhis G, Cosnuau K, Guilmois G, Rumilly E, Blangy E. View: a wheelchair simulator for driving analysis. Assistive technology. 2018 Oct 17.

50. Lam JF, Gosselin L, Rushton PW. Use of virtual technology as an intervention for wheelchair skills training: a systematic review. Archives of physical medicine and rehabilitation. 2018 Nov 1;99(11):2313-41.

51. Guillén-Climent S, Garzo A, Muñoz-Alcaraz MN, Casado-Adam P, Arcas-Ruiz-Ruano J, Mejías-Ruiz M, Mayordomo-Riera FJ. A usability study in patients with stroke using MERLIN, a robotic system based on serious games for upper limb rehabilitation in the home setting. Journal of neuroengineering and rehabilitation. $2021 \mathrm{Dec} ; 18(1): 1-6$.

52. Rozevink SG, van der Sluis CK, Garzo A, Keller T, Hijmans JM. HoMEcare aRm rehabiLItatioN (MERLIN): telerehabilitation using an unactuated device based on serious games improves the upper limb function in chronic stroke. Journal of NeuroEngineering and Rehabilitation. $2021 \mathrm{Dec} ; 18(1): 1-2$.

53. Osuagwu BA, Timms S, Peachment R, Dowie S, Thrussell H, Cross S, Shirley R, Segura-Fragoso A, Taylor J. Home-based rehabilitation using a soft robotic hand glove device leads to improvement in hand function in people with chronic spinal cord injury: a pilot study. Journal of neuroengineering and rehabilitation. 2020 Dec;17(1):1-5.

54. Del Corral T, i Iranzo MÀ, López-de-Uralde-Villanueva I, Martínez-Alejos R, Blanco I, Vilaró J. Effectiveness of a home-based active video game programme in young cystic fibrosis patients. Respiration. 2018;95(2):87-97.

55. Ferre CL, Brandão M, Surana B, Dew AP, Moreau NG, Gordon AM. Caregiver-directed home-based intensive bimanual training in young children with unilateral spastic cerebral palsy: a randomized trial. Developmental Medicine \& Child Neurology. 2017 May;59(5):497-504.

56. Mockler SR, McEwen IR, Jones MA. Retrospective analysis of predictors of proficient power mobility in young children with severe motor impairments. Archives of physical medicine and rehabilitation. 2017 Oct 1;98(10):2034-41.

57. Metts S. An exploratory investigation of deception in close relationships. Journal of Social and Personal relationships. 1989 May;6(2):15979.

\section{Tables}

Table 1a: Characteristics of the study sample 


\begin{tabular}{|c|c|c|c|c|}
\hline Variable $(\mathrm{N}=36)$ & Whole sample & Simulator practice & Power mobile WC & p-value \\
\hline Age (years)- mean (SD) & 10.79 (3.77) & $10.86(3.90)$ & $10.61(3.74)$ & 0.79 \\
\hline \multicolumn{5}{|l|}{ Gender } \\
\hline Boys & $23(64 \%)$ & $10(44 \%)$ & $13(56 \%)$ & 0.49 \\
\hline Girls & $13(36 \%)$ & $8(62 \%)$ & $5(38 \%)$ & \\
\hline \multicolumn{5}{|l|}{ Native language } \\
\hline Hebrew & $25(69 \%)$ & $11(44 \%)$ & $14(56 \%)$ & 0.47 \\
\hline Other & $11(31 \%)$ & $7(64 \%)$ & $4(36 \%)$ & \\
\hline \multicolumn{5}{|l|}{ Education type } \\
\hline Special education & $29(81 \%)$ & $14(48 \%)$ & $15(52 \%)$ & 1.00 \\
\hline Other & $7(19 \%)$ & $4(57 \%)$ & $3(43 \%)$ & \\
\hline \multicolumn{5}{|l|}{ Diagnosis } \\
\hline Cerebral palsy & $32(89 \%)$ & $15(47 \%)$ & $17(53 \%)$ & 0.60 \\
\hline Other & $4(11 \%)$ & $3(75 \%)$ & $1(25 \%)$ & \\
\hline \multicolumn{5}{|l|}{ Walking Ability } \\
\hline No functional walk & $23(64 \%)$ & $11(48 \%)$ & $12(52 \%)$ & 1.00 \\
\hline Therapeutic walk & $13(36 \%)$ & $7(54 \%)$ & $6(46 \%)$ & \\
\hline \multicolumn{5}{|l|}{ Manual WC } \\
\hline Cannot propel manual WC & $25(69 \%)$ & $10(40 \%)$ & $15(60 \%)$ & 0.15 \\
\hline Can propel indoor for short distances & $11(31 \%)$ & $8(73 \%)$ & $3(27 \%)$ & \\
\hline \multicolumn{5}{|l|}{ Practice location } \\
\hline Home & $24(67 \%)$ & $16(67 \%)$ & $8(33 \%)$ & 0.01 \\
\hline Home and school & $12(33 \%)$ & $2(17 \%)$ & $10(83 \%)$ & \\
\hline \multicolumn{5}{|l|}{ LSS levela } \\
\hline Level II & $2(6 \%)$ & $1(50 \%)$ & $1(50 \%)$ & 1.00 \\
\hline Level III & $25(69 \%)$ & $12(48 \%)$ & $13(52 \%)$ & \\
\hline Level IV & $9(25 \%)$ & $5(56 \%)$ & $4(44 \%)$ & \\
\hline \multicolumn{5}{|l|}{ GMFCS $^{b}$} \\
\hline Level III & $1(3 \%)$ & $1(100 \%)$ & $0(0 \%)$ & 0.37 \\
\hline Level IV & $17(53 \%)$ & $9(53 \%)$ & $8(47 \%)$ & \\
\hline Level V & $14(44 \%)$ & $5(36 \%)$ & $9(64 \%)$ & \\
\hline \multicolumn{5}{|l|}{ MACS $^{c}$} \\
\hline Level II & $2(6 \%)$ & $2(100 \%)$ & $0(0 \%)$ & 0.29 \\
\hline Level IV & $23(72 \%)$ & $9(39 \%)$ & $14(61 \%)$ & \\
\hline Level V & $7(22 \%)$ & $4(57 \%)$ & $3(43 \%)$ & \\
\hline \multicolumn{5}{|l|}{ CFCS $^{d}$} \\
\hline Level II & $5(16 \%)$ & $3(60 \%)$ & $2(40 \%)$ & 0.24 \\
\hline Level III & $9(28 \%)$ & $4(44 \%)$ & $5(56 \%)$ & \\
\hline Level IV & $14(44 \%)$ & $8(57 \%)$ & $6(43 \%)$ & \\
\hline
\end{tabular}




\begin{tabular}{|c|c|c|c|c|}
\hline Level V & $4(13 \%)$ & $0(0 \%)$ & $4(100 \%)$ & \\
\hline Brooke $e^{e}$ & & & & na \\
\hline Participant 13 & 4 & 4 & - & \\
\hline Participant 16 & 2 & 2 & - & \\
\hline Participant 20 & 4 & 4 & - & \\
\hline Participant 35 & 2 & - & 2 & \\
\hline Vignos $^{f}$ & & & & $\mathrm{Na}$ \\
\hline Participant 13 & 9 & 9 & - & \\
\hline Participant 16 & 9 & 9 & - & \\
\hline Participant 20 & 7 & 7 & - & \\
\hline Participant 35 & 9 & - & 9 & \\
\hline
\end{tabular}

$a=$ Inter-rater reliability $(K=0.60)$; Test retest reliability $(K=0.55)[31]$.

$b=$ Inter-rater reliability $(K=0.84, p<0.0001)[32,33]$

C= Inter-rater reliability (ICC) between therapists was 0.97 (95\% confidence interval 0.96-0.98)[34]

$\mathrm{d}=$ Inter-rater reliability among professionals $\mathrm{K}=0.66(95 \%$ confidence interval $\mathrm{Cl}=0.55-0.78)$; Test retest reliability $\mathrm{K}=0.82(95 \% \mathrm{Cl}=0.74-$ 0.90)[35].

e= Inter-rater reliability ICC $=0.87$; Test retest reliability ICC $=0.92$ [37]

$\mathrm{f}=$ Inter-rater reliability ICC $=0.96$; Test retest reliability ICC $=0.99$ [37]

Table 1b Baseline outcome measure scores

\begin{tabular}{lllll} 
Outcome Measure & Whole sample & Simulator practice & Power mobile WC & p-value \\
\hline Pre-practice PMP score-median (IQR)* & $3.00(0.50-4.00)$ & $2.00(1.18-3.31)$ & $1.62(0.00-2.68)$ & 0.22 \\
\hline Pre-practice ALP score-median (IQR)* & $5.00(3.00-6.12)$ & $5.00(3.00-6.00)$ & $4.00(2.25-4.75)$ & 0.22 \\
\hline Pre-practice PM-PT score-median (IQR)* & $2.25(1.00-4.00)$ & $1.50(1.00-3.00)$ & $2.00(1.00-200)$ & 0.58
\end{tabular}

* The pre-practice measures - PMP, ALP and PM-MT - were calculated on 30 children who had pre- and post- data.

Table 2: Pre- and post-practice differences 


\begin{tabular}{|c|c|c|c|c|c|c|c|}
\hline Assessment & Group & $\begin{array}{l}\text { Time- } \\
\text { point }\end{array}$ & $\begin{array}{l}\text { Median } \\
\text { (Inter-quartile } \\
\text { range) }\end{array}$ & $\begin{array}{l}\text { Practice } \\
\text { effect }\end{array}$ & $\begin{array}{l}\text { Group } \\
\text { effect }\end{array}$ & $\begin{array}{l}\text { Simple effects: } \\
\text { practice } \\
\text { (pre-practice: post- } \\
\text { practice) }\end{array}$ & $\begin{array}{l}\text { Simple effects: } \\
\text { group } \\
\text { (simulator: } \\
\text { powered WC) }\end{array}$ \\
\hline \multirow[t]{6}{*}{$\mathrm{PMP}^{\mathrm{a}}$} & Simulator & $\begin{array}{l}\text { Pre- } \\
\text { practice }\end{array}$ & $\begin{array}{l}2.00(1.18- \\
3.31)\end{array}$ & $\begin{array}{l}z=-3.62 \\
p<0.001\end{array}$ & & & \\
\hline & & & & & $\begin{array}{l}z=-0.06 \\
p=0.22\end{array}$ & $p=0.04$ & $z=-1.26, p=0.22$ \\
\hline & & $\begin{array}{l}\text { Post- } \\
\text { Practice }\end{array}$ & $\begin{array}{l}2.50(1.00- \\
5.00)\end{array}$ & & & & \\
\hline & $\begin{array}{l}\text { Powered } \\
\text { WC }\end{array}$ & $\begin{array}{l}\text { Pre- } \\
\text { practice }\end{array}$ & $\begin{array}{l}1.62(0.00- \\
2.68)\end{array}$ & & & $z=-2.95$ & $\mathrm{U}=94.50$ \\
\hline & & & & & & $p=0.003$ & $z=-0.74, p=0.47$ \\
\hline & & $\begin{array}{l}\text { Post- } \\
\text { Practice }\end{array}$ & $\begin{array}{l}2.00(1.12- \\
4.37)\end{array}$ & & & & \\
\hline \multirow[t]{6}{*}{$A L P^{b}$} & Simulator & $\begin{array}{l}\text { Pre- } \\
\text { practice }\end{array}$ & $\begin{array}{l}5.00(3.00- \\
6.00)\end{array}$ & $\begin{array}{l}z=-2.39 \\
p=0.02\end{array}$ & & $z=-0.98$ & $\mathrm{U}=82.50$ \\
\hline & & & & & $\begin{array}{l}z=-0.92 \\
p=0.38\end{array}$ & $p=0.33$ & $z=-1.24, p=0.22$ \\
\hline & & $\begin{array}{l}\text { Post- } \\
\text { Practice }\end{array}$ & $\begin{array}{l}4.00(3.00- \\
7.00)\end{array}$ & & & & \\
\hline & $\begin{array}{l}\text { Powered } \\
\text { WC }\end{array}$ & $\begin{array}{l}\text { Pre- } \\
\text { practice }\end{array}$ & $\begin{array}{l}4.00(2.25- \\
4.75)\end{array}$ & & & & $U=101.00$ \\
\hline & & & & & & $p=0.03$ & $z=-0.46, p=0.67$ \\
\hline & & $\begin{array}{l}\text { Post- } \\
\text { Practice }\end{array}$ & $\begin{array}{l}5.00(3.00- \\
7.00)\end{array}$ & & & & \\
\hline \multirow[t]{6}{*}{$\mathrm{PM}-\mathrm{PT}^{\mathrm{c}}$} & Simulator & $\begin{array}{l}\text { Pre- } \\
\text { practice }\end{array}$ & $\begin{array}{l}1.50(1.00- \\
3.00)\end{array}$ & $\begin{array}{l}z=-3.58 \\
p<0.001\end{array}$ & $U=93.00$ & $z=-2.41$ & $\mathrm{U}=98.00$ \\
\hline & & & & & $\begin{array}{l}z=-0.81 \\
p=0.45\end{array}$ & $p=0.01$ & $z=-0.62, p=0.58$ \\
\hline & & $\begin{array}{l}\text { Post- } \\
\text { Practice }\end{array}$ & $\begin{array}{l}3.00(1.00- \\
5.00)\end{array}$ & & & & \\
\hline & $\begin{array}{l}\text { Powered } \\
\text { WC }\end{array}$ & $\begin{array}{l}\text { Pre- } \\
\text { practice }\end{array}$ & $\begin{array}{l}2.00(1.00- \\
2.00)\end{array}$ & & & $z=-2.68$ & $\mathrm{U}=101.00$ \\
\hline & & & & & & $p=0.007$ & $z=-0.48, p=0.67$ \\
\hline & & $\begin{array}{l}\text { Post- } \\
\text { Practice }\end{array}$ & $\begin{array}{l}3.00(1.00- \\
4.75)\end{array}$ & & & & \\
\hline
\end{tabular}

$a=$ Inter-rater reliability $(r=0.99, p<0.01)$; intra-rater reliability $(r=0.99, p<0.01)[38,39]$

$b=$ Inter-rater reliability $(K=0.83-0.87)[39,40]$

$\mathrm{C}=$ Inter-rater reliability $\mathrm{ICC}_{2,1}$ mean= 0.89; Intra-rater $\mathrm{ICC}_{2,1}$ mean= 0.93 [39]

Table 3 User experience 


\begin{tabular}{|c|c|c|c|c|c|c|c|c|c|}
\hline $\begin{array}{l}\text { Question/ } \\
\text { Mode }\end{array}$ & actice & Age & $\begin{array}{l}\text { 1) To what } \\
\text { extent did your } \\
\text { child enjoy the } \\
\text { practice on the } \\
\text { simulator/chair? }\end{array}$ & $\begin{array}{l}\text { 2) To } \\
\text { what } \\
\text { extent, } \\
\text { you the } \\
\text { parent, } \\
\text { enjoy } \\
\text { the } \\
\text { practice } \\
\text { time? }\end{array}$ & $\begin{array}{l}\text { 3) To what } \\
\text { extent do } \\
\text { you think } \\
\text { your child } \\
\text { progressed } \\
\text { in his/her } \\
\text { practice in } \\
\text { comparison } \\
\text { to the } \\
\text { amount of } \\
\text { time } \\
\text { invested? }\end{array}$ & $\begin{array}{l}\text { 4) If your } \\
\text { child } \\
\text { practiced } \\
\text { on the } \\
\text { simulator- } \\
\text { to what } \\
\text { extent did } \\
\text { they have } \\
\text { unpleasant } \\
\text { symptoms } \\
\text { during } \\
\text { training } \\
\text { ("car } \\
\text { sickness”, } \\
\text { nauseous, } \\
\text { instability } \\
\text { )? }\end{array}$ & $\begin{array}{l}\text { 5) As } \\
\text { parents- } \\
\text { how } \\
\text { important } \\
\text { was the } \\
\text { practice in } \\
\text { the process } \\
\text { of obtaining } \\
\text { independent } \\
\text { mobility? }\end{array}$ & $\begin{array}{l}\text { 6) How } \\
\text { important } \\
\text { did your } \\
\text { child think } \\
\text { the practice } \\
\text { was to } \\
\text { obtain } \\
\text { independent } \\
\text { mobility }\end{array}$ & $\begin{array}{l}\text { 7) } \\
\text { Compliance- } \\
\text { your child } \\
\text { was asked } \\
\text { to practice } \\
\text { at least } 4 \\
\text { times a } \\
\text { week for } 20 \\
\text { minutes- } \\
\text { Was your } \\
\text { child's } \\
\text { practice } \\
\text { time as per } \\
\text { requested? }\end{array}$ \\
\hline \multirow[t]{3}{*}{ Chair } & Mean & 11.7 & 4.6 & 4.5 & 4.4 & $\mathrm{~N} / \mathrm{A}$ & 4.5 & 4.3 & 3.73 \\
\hline & $\mathrm{N}$ & 15.0 & 15.0 & 15.0 & 15.0 & $\mathrm{~N} / \mathrm{A}$ & 15.0 & 15.0 & 15.0 \\
\hline & $\begin{array}{l}\text { Std. } \\
\text { Deviation }\end{array}$ & 3.3 & 0.7 & 0.7 & 1.1 & $\mathrm{~N} / \mathrm{A}$ & 0.7 & 0.8 & 1.3 \\
\hline \multirow[t]{4}{*}{ Simulator } & & 10.7 & 4.3 & 4.2 & 3.8 & 4.6 & 4.7 & 4.0 & 3.0 \\
\hline & Mean & & & & & & & & \\
\hline & $N$ & 6.0 & 6.0 & 6.0 & 6.0 & 6.0 & 6.0 & 6.0 & 6.0 \\
\hline & $\begin{array}{l}\text { Std. } \\
\text { Deviation }\end{array}$ & 3.0 & 0.8 & 1.2 & 1.5 & 0.7 & 0.5 & 0.9 & 1.4 \\
\hline \multirow[t]{4}{*}{ Total } & & 11.4 & 4.5 & 4.4 & 4.2 & & 4.5 & 4.2 & 3.5 \\
\hline & Mean & & & & & & & & \\
\hline & $N$ & 21.0 & 21.0 & 21.0 & 21.0 & & 21.0 & 21.0 & 21.0 \\
\hline & $\begin{array}{l}\text { Std. } \\
\text { Deviation }\end{array}$ & 3.2 & 0.8 & 0.9 & 1.2 & & 0.7 & 0.8 & 1.4 \\
\hline
\end{tabular}

\section{Figures}


a.

b.

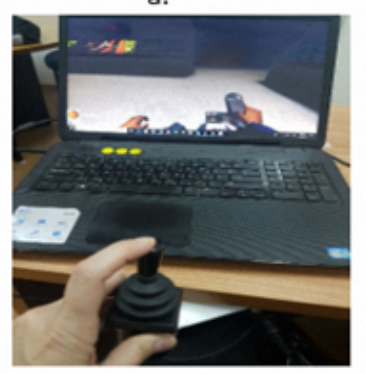

c.
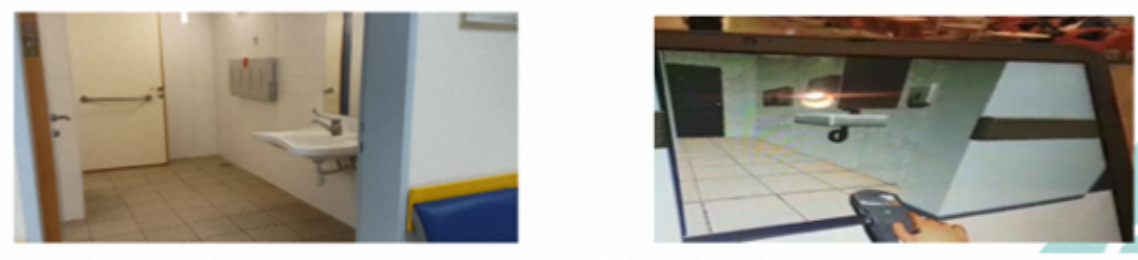

d.

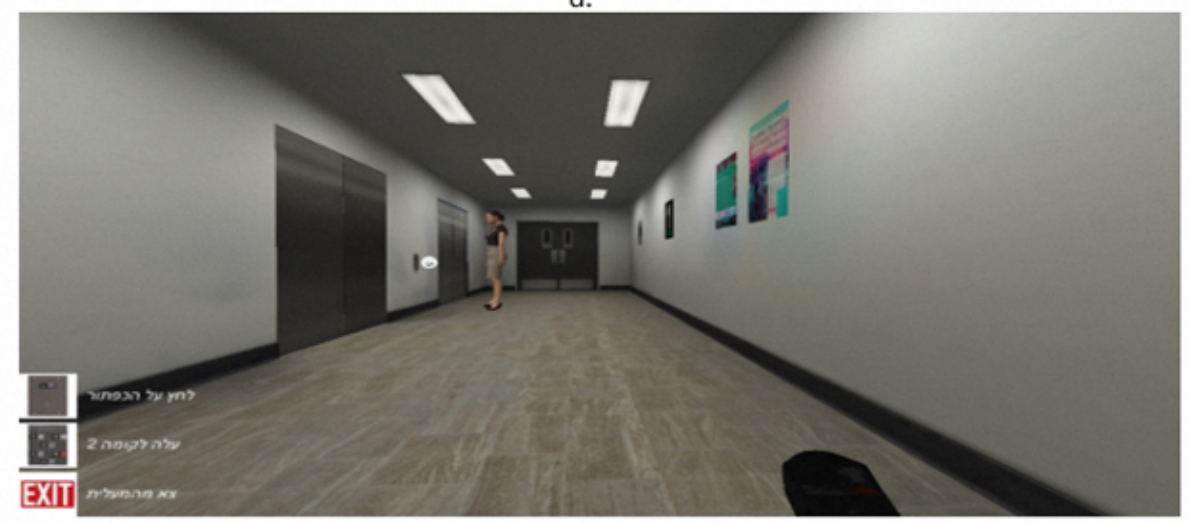

Figure 1

a. MiWe-C setup; b. Real bathroom; c. MiWe-C (simulated) bathroom; d MiWe-C (simulated) approach to elevator 


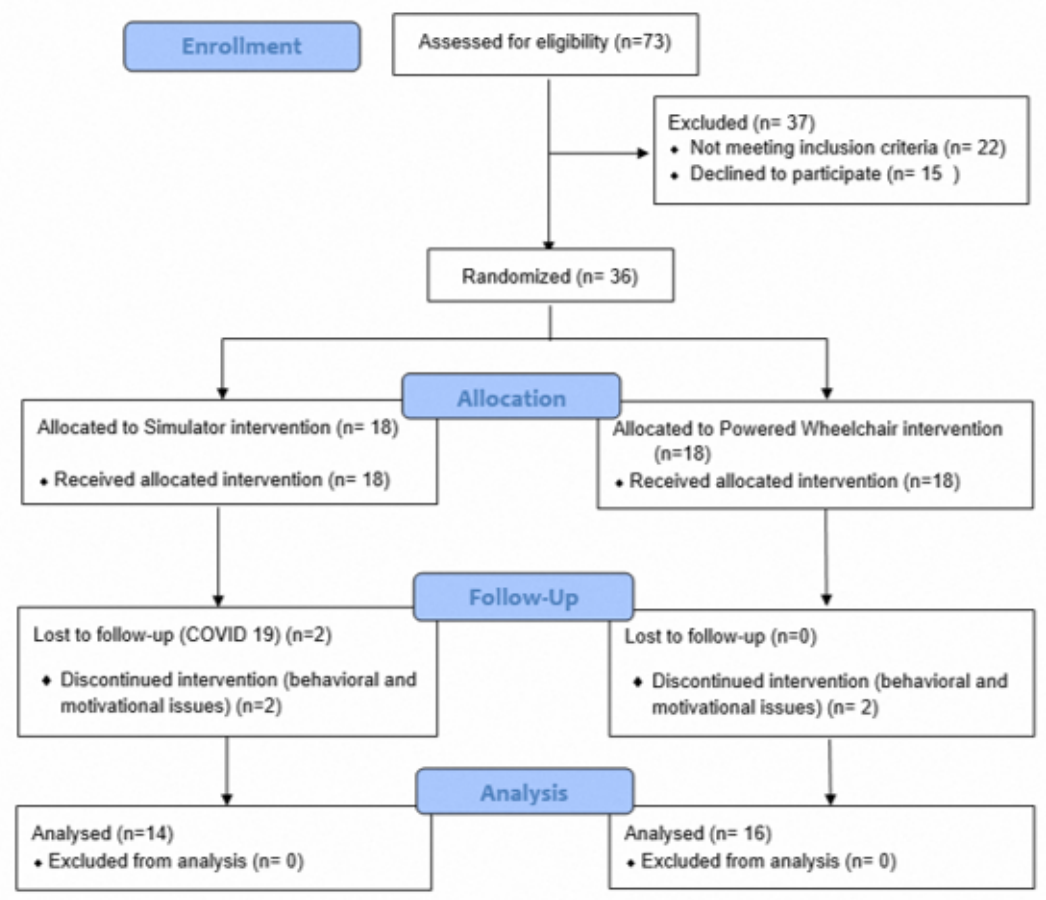

Figure 2

Consort Diagram $(n=36)$

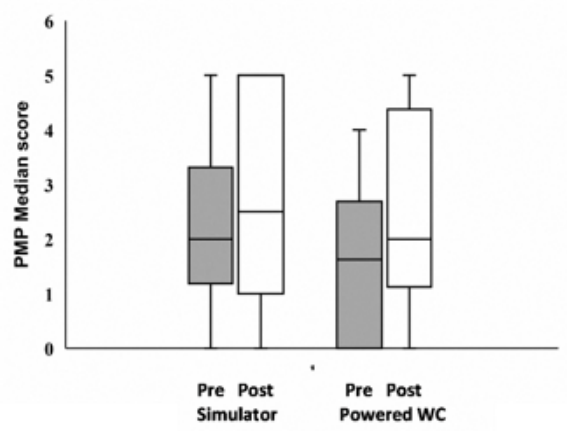

(a)

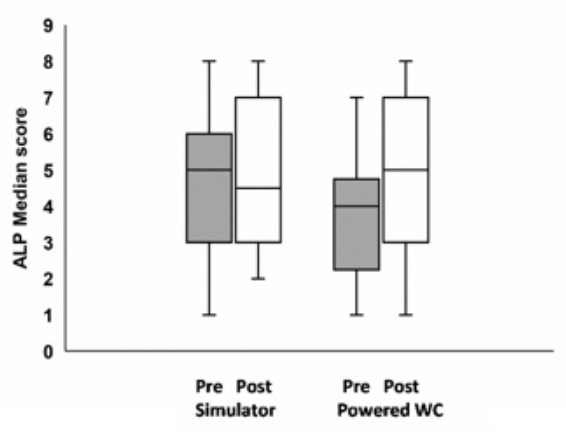

(b)

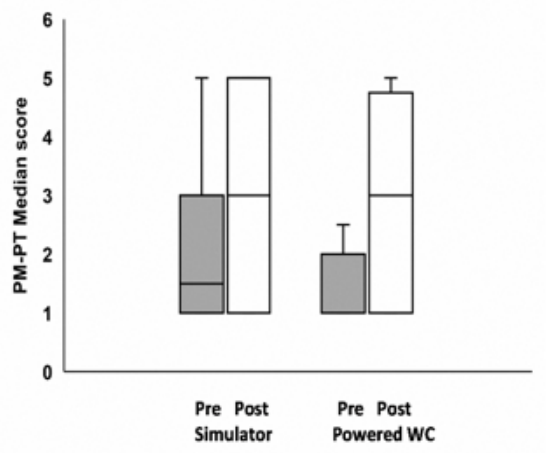

(c)

\section{Figure 3}

Differences between the median pre- and post-intervention scores of the (a) PMP, (b) ALP and (c) PM-PT for the simulator practice group (grey) and the powered mobility group (white). The plots represent the inter-quartile ranges. a: PMP score (median) b: ALP score (median) c: PM-PT score (median) 